\title{
Maternal Isobutyl-Paraben Exposure decreases the Plasma Corticosterone Level in Dams and Sensitivity to Estrogen in Female Offspring Rats
}

\author{
Maiko KAWAGUCHI ${ }^{1)}$, Kaori MOROHOSHI ${ }^{2) * *}$, Junya MASUDA ${ }^{1)}$, Gen WATANABE ${ }^{3,4)}$, \\ Masatoshi MORITA ${ }^{5,6)}$, Hideki IMAI ${ }^{7)}$, Kazuyoshi TAYA ${ }^{3,4)}$ and Toshiyuki HIMI ${ }^{1) *}$ \\ ${ }^{1)}$ Faculty of Pharmacy and Research Institute of Pharmaceutical Science, Musashino University, 1-1-20 Shinmachi, Nishitokyo, Tokyo \\ 202-8585, 2) Biological Risk Assessment Section, Environmental Health Sciences Division, National Institute for Environmental Studies \\ 16-2 Onogawa, Tsukuba, Ibaraki 305-8506, ${ }^{3}$ Laboratory of Veterinary Physiology, Department of Veterinary Medicine, Faculty of \\ Agriculture, Tokyo University of Agriculture and Technology, 3-5-8 Saiwai-cho, Fuchu, Tokyo 183-8509, ${ }^{4}$ Department of Basic \\ Veterinary Science, The United Graduate School of Veterinary Sciences, Gifu University, 1-1 Yanagido, Gifu, Gifu 501-1193, \\ ${ }^{5}$ Research Center for Environmental Risk, National Institute for Environmental Studies, 16-2 Onogawa, Tsukuba, Ibaraki 305-8506, \\ ${ }^{6)}$ Department of Bioresources, Faculty of Agriculture, Ehime University, 3-5-7 Tarumi, Matsuyama, Ehime 790-8566 and \\ ${ }^{7)}$ Division of Environmental Health Sciences, Department of Social Medicine, Faculty of Medicine, University of Miyazaki, 5200 Kihara, \\ Kiyotake, Miyazaki 889-1692, Japan
}

(Received 20 January 2009/Accepted 12 March 2009)

ABSTRACT. Isobutyl-paraben (IBP), a widely used preservative in a variety of foods, shows high comparative binding affinity to estrogen receptors. Here, we examined the effects of maternal exposure of rats to IBP on plasma hormone concentrations and organ weights in dams, ratio of male pups, anogenital distance, organ weights and plasma hormone concentrations in offspring, puberty, estrous cycle and response of organ weight and plasma hormone concentrations to estrogen in adult female offspring, and reproductive and adrenal function in adult male offspring, all of which are under developmental estrogen regulation, to clarify the estrogenic effects of IBP during gestation and lactation on the endocrine systems of dams and offspring. While maternal exposure of IBP decreased the plasma corticosterone concentration and increased the uterus weight in dams and increased uterine sensitivity to estrogen in adult female offspring, the other indices examined were largely unaffected by the present treatment. Even though these results indicate little sign of endocrine disrupting effects for IBP, the existence of activity may be a matter of concern due to the possible impact on the health of future generations. KEY WORDS: corticosterone, estrogen, isobutyl-paraben, maternal, rat.

Parabens have been the most widely used preservatives for more than 50 years in a variety of food, cosmetic and pharmaceutical products $[2,4,23]$ due to their broad spectrum of activity, inertness, low cost and long history of safe use. They have been detected in human urine collected from a demographically diverse group of male and female adults with no known exposure to parabens, suggesting that the majority of people have already been exposed to them [32]. Several studies indicate that exposure to parabens modulates the endocrine system and thus may have harmful consequences on animal and human health $[4,7,13,22,28]$ because of the estrogenic activities of these compounds in vitro and in vivo [7, 24], and anti-estrogenic activity in human skin has been shown [22]. In addition, exposure to parabens during adult periods has been implicated in reduction of the male reproductive system in rodents $[7,19,20$, 21], whereas a recent study could not confirm these effects [10]. On the other hand, investigation of the effects of exposure to parabens during gestation to lactation is important because exposure to estrogenic chemicals during develop-

\footnotetext{
* CORRESPONDENCE TO: Himi, T., Faculty of Pharmacy and Research Institute of Pharmaceutical Science, Musashino University, 1-120 Shinmachi, Nishitokyo, Tokyo 202-8585, Japan. e-mail: to-himi@musashino-u.ac.jp

**Present address: Morohoshi, K., Mitsubishi Chemical Safety Institute Ltd., Yokohama Laboratory, 1000 Kamoshida-cho, Aoba, Yokohama, Kanagawa 227-0033, Japan.
}

ment could be a contributory factor in the rising incidence of endocrine dysfunction of offspring later in life $[3,5,14,16$, $26]$. But, the effects of early exposure to parabens are not consistent in previous reports $[6,13,27,28]$. Furthermore, the effects of early exposure to parabens on the endocrine system in adult female offspring, except for vaginal opening and intact uterus weight [13], are unclear. Therefore, we examined the effects of maternal exposure to parabens on several aspects of the endocrine system in male and female offspring, especially the effects on plasma hormone concentrations and semen analysis. In addition, we examined the effects of exposure to IBP on maternal endocrine systems that are affected by estrogen and influence development of offspring. Isobutyl-paraben (IBP), belonging to the family of parabens, shows high comparative binding affinity to estrogen receptors (ER) $\alpha$ and $\beta$ [7] and comparative potency for estrogen agonistic activities in a yeast twohybrid assay [18]. Here, we examined the effects of maternal exposure through gestation to lactation of rats to IBP on the plasma hormone concentrations and organ weights in dams, ratio of male pups, anogenital distance (AGD), organ weights and plasma hormone concentrations in offspring, puberty, the estrus cycle and response of organ weight and plasma hormone concentrations to estrogen in adult female offspring, and reproductive and adrenal function in adult male offspring, all of which are under developmental estro- 
gen regulation, to clarify the estrogenic effects of IBP during gestation and lactation on the endocrine systems of dams and offsprings.

\section{MATERIALS AND METHODS}

Sprague Dawley rats were obtained from Charles River Laboratories Japan, Inc. (Ibaraki, Japan) and maintained under conditions of controlled lighting (lights on, 07.00$19.00)$, temperature $\left(22.5 \pm 0.5^{\circ} \mathrm{C}\right)$ and humidity $(55 \pm$ 10\%). Food (CE2, CLEA Japan, Inc., Tokyo, Japan) and distilled water in glass bottles were available ad libitum. Adult rats and nursing dams with pups were housed one adult per stainless steel cage lined with paper bedding (Paper Clean, Japan SLC, Inc., Shizuoka, Japan). The animals were maintained and used according to the guidelines of our institutional Animal Care Committee and the National Institute for Environmental Studies Animal Care and Use Committee.

Thirteen-week-old virgin female rats were paired with 11-week-old fertile male rats from the evening of proestrus to the morning of estrus. Female rats that showed a regular 4-d estrous cycle 2 weeks prior to mating were used in the present study. Mating was verified by the presence of sperm in vaginal smears (gestational day 0; GD0). Pups were counted and culled on PD3 (PD0=day of birth) to leave four males and four females per litter. These pups were kept with their respective dams until weaning on PD21. Three weeks before mating, a 20-mm long Silastic capsule, $2 \mathrm{~mm}$ i.d., 3 mm o.d. (Kaneka Medix Co., Osaka, Japan) that was either filled with IBP (isobutyl 4-hydroxybenzoate; Tokyo Chemical Industry Co., Ltd., Tokyo, Japan) or unfilled (control) was implanted into the female rats under the skin of the flank region. To determine in vitro release of IBP from the Silastic capsules, the capsule was incubated in $120 \mathrm{~m} l$ saline at $37^{\circ} \mathrm{C}$ for $42 \mathrm{~d}$. The incubation media were periodically analyzed using high-performance liquid chromatography (HPLC, Shimadzu, Kyoto, Japan) to determine the amount of IBP released. Briefly, saline was isocratically fractionated at $1.0 \mathrm{ml} / \mathrm{min}$ on an analytical reverse phase $\mathrm{C}_{18}$ column using $40 \%$ acetonitrile and phosphate buffer. The elution profiles of IBP were monitored at $254 \mathrm{~nm}$. The amounts of IBP in the fractionated media were determined based on peak heights after calibration with known amounts of IBP. Silastic capsules filled with IBP released about $4.36 \mathrm{mg} / \mathrm{ll}$ day IBP into $37^{\circ} \mathrm{C}$ saline.

For AGD, 4 males and 4 females from each litter were examined at day 7. Dams at weaning, one male and one female weanling per litter at 3 weeks of age and one male per litter at 12 weeks of age were decapitated at approximately $11.00 \mathrm{hr}$. After decapitation, trunk blood, the adrenal gland, pituitary, testis and uterus were collected, and the organs were weighed. The right testes of 12-week-old males were decapsulated, placed in a measured volume of saline and frozen at $-20^{\circ} \mathrm{C}$ for measurement of total sperm number.

To confirm adrenal function disturbances during IBP exposure, 12-week-old male offspring were stressed by 2-hr immobilization in small disposable restrainers (DecapiCone; Braintree Scientific, Inc., Braintree, MA, U.S.A.; one offspring per litter). The rats were immobilized from approximately $9.00 \mathrm{hr}$ until decapitation at $11.00 \mathrm{hr}$. After decapitation, trunk blood and the adrenal gland were collected.

One female from each litter was monitored daily for vaginal opening from weaning and for estrus cycle at 7-weeks of age. Other 7-week-old female offspring were ovariectomized (OVX), and a 15-mm long Silastic capsule, $2 \mathrm{~mm}$ i.d., $3 \mathrm{~mm}$ o.d. that was either filled with $20 \% 17-\beta$ estradiol (E2; Sigma Chemical Co., St Louis, MO, U.S.A.) in cholesterol (Sigma Chemical Co.) or cholesterol (one offspring per litter) was then implanted under the skin of the flank region. To determine the sensitivity to estrogen resulting from developmental IBP treatment, E2-implanted OVX rats were decapitated between 16.00 and $17.00 \mathrm{hr}$ at 12 weeks of age, trunk blood, the pituitary and the uterus were collected and the organs were weighed 5 weeks after E2 implantation. Six weeks after the surgery (13 weeks of age), blood samples were collected from the cholesterol implanted OVX rats via the jugular vein under ether anesthesia between 15.00 and $17.00 \mathrm{hr}$. Blood samples were also collected from 17-weekold female offspring (one per litter) via the jugular vein under ether anesthesia at around $14.00 \mathrm{hr}$ at diestrus.

All blood samples were collected into plastic tubes containing heparin to prevent clotting. The samples were stored in ice and centrifuged at $3,000 \times \mathrm{g}$ for $10 \mathrm{~min}$ at $4^{\circ} \mathrm{C}$ immediately after collection. The resulting plasma was stored at $-20^{\circ} \mathrm{C}$ for later analysis of the estradiol, testosterone, luteinizing hormone (LH), follicle-stimulating hormone (FSH), prolactin, ir-inhibin, total triiodothyronine (TT3), total thyroxine (TT4) and corticosterone levels.

Changes in spermatogenesis in the testis were evaluated as described previously [17]. In brief, one drop of extracted emulsion was placed on a hematocytometer after sufficient dilution with saline, and the sperm heads were then counted under a phase-contrast microscope. Fourteen-week-old male rats were kept for an extended period of time in a chamber containing ether vapor to deliver deep anesthesia and were exsanguinated by heart puncture. The right epididymis was then removed. The sperm motility parameters were obtained using a C. IMAGING computer-assisted sperm motion analysis system (Compix Inc, Cranberry Township, PA, U.S.A.) as described previously [9]. In brief, sperm were incubated at $37^{\circ} \mathrm{C}$ for $3 \mathrm{~min}$ in one drop of caudal epididymis fluid. After incubation, an aliquot of this solution was diluted 10 - to 20-fold with the medium, and 10 $\mu l$ was placed into a $50-\mu \mathrm{m}$ deep microcell-HAC chamber (Conception Technologies, San Diego, CA, U.S.A.). Analyses of motile characteristics were performed on at least 200 cells for each sample, and sperm motion, as viewed on an Olympus microscope $(4 \times$, pseudodark field optics $)$ with a stage warmer $\left(37^{\circ} \mathrm{C}\right)$ (MP-10DM, Kitazato Supply Co., Ltd., Shizuoka, Japan), was measured using a C. IMAGING system. The C. IMAGING system settings were as follows: 
frames analyzed $=15$, framing rate $=30$, maximum velocity $=1200 \mu \mathrm{m} / \mathrm{s}$, threshold velocity $=45 \mu \mathrm{m} / \mathrm{s}$, minimum linearity for mean amplitude of lateral head displacement $(\mathrm{ALH})=3.5$, pixel scale $=3.26 \mu \mathrm{m} / \mathrm{pixel}$, maximum average number of cells/field $=30$ and cell size range $=350$ 1600 pixels. The following characteristics were analyzed: the percentage of motile spermatozoa, curvilinear velocity (the total distance traveled divided by the total time the cell was tracked), straight velocity (straight line distance), ALH (deviation of the sperm head from the mean trajectory), beat/cross frequency, linearity (ratio of the straight line distance to the actual tracked distance) and the percentage of circular cells.

Plasma was analyzed for rat $\mathrm{LH}, \mathrm{FSH}$ and prolactin by radioimmunoassay using a rat kit from the National Institute of Diabetes and Digestive and Kidney Disease (NIDDK; Bethesda, MD, U.S.A.). The iodinated preparations were rat-LH-I-7, rat-FSH-I-7 and rat-prolactin-I-6; the antisera used were anti-rat LH-S-10, anti-rat FSH-S-11 and anti-rat prolactin-S-9. The results were expressed as rat LH RP-2, rat FSH RP-2 and rat prolactin RP-3. The intra-assay coefficients of variation (CVs) were $8.9 \%$ for $\mathrm{LH}, 4.4 \%$ for $\mathrm{FSH}$ and $2.8 \%$ for prolactin. The plasma concentrations of $\mathrm{LH}$, $\mathrm{FSH}$ and prolactin were measured in a single assay.

The plasma concentrations of ir-inhibins were measured using a rabbit antiserum against purified bovine inhibin and ${ }^{125}$ I-labeled 32-kDa bovine inhibin, as described previously [8]. Estradiol was measured by double-antibody RIA using ${ }^{125}$ I-labeled radioligands as described previously [29]. Antisera against estradiol (GDN 244; [15] were kindly provided by Dr. G. D. Niswender (Colorado State University, Fort Collins, CO, U.S.A.). The intra-assay CVs were $4.1 \%$ for estradiol and $8.8 \%$ for ir-inhibin. The plasma concentrations of estradiol and ir-inhibin were measured in a single assay.

Plasma testosterone, TT3, TT4 and corticosterone were determined by radioimmunoassay using commercial kits (Diagnostic Products, Los Angeles, CA, U.S.A.). The intraassay CVs were $6.3 \%$ for testosterone, $3.7 \%$ for TT3, $2.7 \%$ for TT4 and $9.8 \%$ for corticosterone. The plasma concentrations of testosterone, TT3, TT4 and corticosterone were measured in a single assay.

All results are expressed as means \pm S.E. When data from only two groups were available, the Mann-Whitney $U$ test was applied. If the Mann-Whitney $U$ test was not significant, the Student's $t$-test was applied after confirming the equality of variance by the $F$-test for two samples. For multiple comparisons, we used 2-way analysis of variance (ANOVA). Where appropriate, Fisher's post hoc tests were utilized to determine group differences. Statistical differences were considered significant when $p<0.05$. All calculations were performed using the StatView 5.0J software for Windows (SAS Inc., Cary, NC, U.S.A.).

\section{RESULTS}

No overt signs of toxicity were noted in this study. There were no observed effects of IBP exposure on litter size (control litter, $13.1 \pm 0.6$; IBP-treated litter, $15.3 \pm 1.0)$ or the ratio of male pups (control litter, $46.1 \pm 7.6 \%$; IBP-treated litter, $46.0 \pm 3.8 \%$ ).

As shown in Table 1, the plasma corticosterone concentrations of the IBP-treated dams decreased significantly to about 3.5 times that of the control group at weaning. In addition, IBP treatment significantly affected the weight of the dam's uterus, though there was no difference in the weight of the pituitary (Table 1). None of the other plasma hormone concentrations or weights of organs of dams and weanlings were affected by the treatment (Table 1). There were no observed effects of IBP treatment on AGD or vaginal opening (Table 2).

The weight of the uterus was significantly lower in the IBP-exposed OVX + E2 female offspring, compared with the control at 12 weeks of age, though there was no difference in the weight of the pituitary and the plasma concentration of E2 due to the implanted Silastic capsule (Table 3). There were no observed effects of IBP treatment on the plasma concentrations of $\mathrm{LH}, \mathrm{FSH}$ and prolactin in the OVX and OVX + E2 female offspring. In the intact females, IBP exposure did not alter the estrus cycle (Table 2) or the plasma concentrations of E2 and LH during diestrus (Table 3).

The plasma levels of corticosterone increased in the 12week-old male offspring in the IBP-treated and control groups $2 \mathrm{hr}$ after immobilization (Table 4). There were no observed effects of IBP treatment on the plasma concentrations of corticosterone and PRL with or without immobilization stress. There were no observed effects of IBP treatment on other plasma hormones or weights of the pituitary and testis in male offsprings. There was no significant difference in total sperm number or the results of the sperm motility analysis between the control and IBP-treated male offspring, except for the fact that BCF was increased.

\section{DISCUSSION}

Maternal exposure to IBP in rats caused pronounced decreases in plasma corticosterone levels and increases in uterus weight in dams at weaning. Previous studies show that estradiol treatment elevates plasma corticosterone levels in OVX rats [1] and that estradiol stimulates adrenocortical H295R cell proliferation [12]. Therefore, low levels of corticosterone in the IBP-exposed dams are due, in part, to inhibition of endogenous estrogen activity because maternal exposure to IBP did not affect the plasma levels of endogenous estrogen. Contrary to corticosterone, the estrogenic activity of IBP may cause an increase in the weights of the uterus and pituitary in IBP-exposed dams. On the other hand, the weights of the pituitary and adrenal, gonadotropin and thyroid hormones, all of which are effected by estrogen, were unaffected by maternal exposure to IBP. These results show that the uterus and corticosterone are highly sensitive to IBP exposure.

In a previous study, decreased levels of maternal gluco- 
Table 1. Organ weights and plasma hormone concentrations in dams and weanlings after exposure to isobutyl-paraben (IBP) during gestation and lactation

\begin{tabular}{|c|c|c|}
\hline & Control & IBP \\
\hline \multicolumn{3}{|c|}{ Plasma hormone concentrations } \\
\hline \multicolumn{3}{|l|}{$\mathrm{LH}(p \mathrm{~g} / \mathrm{m} l)$} \\
\hline Dam & $0.46 \pm 0.20(8)$ & $0.22 \pm 0.04$ \\
\hline Male weanling & $0.039 \pm 0.013(6)$ & $0.074 \pm 0.047(6$ \\
\hline Female weanling & $1.201 \pm 0.590(8)$ & $0.492 \pm 0.191(6$ \\
\hline \multicolumn{3}{|l|}{$\mathrm{FSH}(p \mathrm{~g} / \mathrm{m} l)$} \\
\hline Dam & $1.31 \pm 0.35(8)$ & $2.32 \pm 1.03(7)$ \\
\hline \multicolumn{3}{|l|}{ Corticosterone $(n \mathrm{~g} / \mathrm{m} l)$} \\
\hline Dam & $476.0 \pm 48.1(7)$ & $138.3 \pm 56.2(5)^{*}$ \\
\hline Male weanling & $187.6 \pm 51.0(7)$ & $132.9 \pm 24.2(7)$ \\
\hline Female weanling & $162.6 \pm 23.2(8)$ & $214.0 \pm 50.4(7)$ \\
\hline \multicolumn{3}{|l|}{$\mathrm{E} 2(\mathrm{pg} / \mathrm{m} l)$} \\
\hline Dam & $13.63 \pm 5.13(8)$ & $13.72 \pm 2.64(7)$ \\
\hline Female weanling & $2.60 \pm 0.78(6)$ & $2.63 \pm 0.85$ \\
\hline \multicolumn{3}{|l|}{ Testosterone $(n \mathrm{~g} / \mathrm{m} l)$} \\
\hline Male weanling & $9.80 \pm 2.30(7)$ & $5.63 \pm 0.78(7)$ \\
\hline \multicolumn{3}{|l|}{ ir-Inhibin $(\mathrm{pg} / \mathrm{m} l)$} \\
\hline Male weanling & $1.57 \pm 0.12(5)$ & $1.55 \pm 0.13(6)$ \\
\hline \multicolumn{3}{|l|}{$\mathrm{TT} 4(\mu \mathrm{g} / \mathrm{m} l)$} \\
\hline Dam & $240 \pm 16(8)$ & $252 \pm 30(7)$ \\
\hline Male weanling & $331 \pm 22(7)$ & $291 \pm 16(7)$ \\
\hline Female weanling & $295 \pm 15(8)$ & $269 \pm 20(7)$ \\
\hline \multicolumn{3}{|l|}{ ТT3 $(\mu \mathrm{g} / \mathrm{m} l)$} \\
\hline Dam & $8.8 \pm 0.6(8)$ & $9.17 \pm 1.1(7)$ \\
\hline Male weanling & $13.6 \pm 0.3(5)$ & $12.6 \pm 0.8(6)$ \\
\hline Female weanling & $12.5 \pm 1.0$ & $12.4 \pm 0.7(6)$ \\
\hline \multicolumn{3}{|l|}{ Organ weight } \\
\hline \multicolumn{3}{|l|}{ Pituitary weight (mg) } \\
\hline Dam & $15.6 \pm 0.4(8)$ & $18.1 \pm 2.1(7)$ \\
\hline Male weanling & $2.50 \pm 0.18(7)$ & $3.14 \pm 0.82(7)$ \\
\hline Female weanling & $2.89 \pm 0.16(8)$ & $3.27 \pm 0.27(7)$ \\
\hline \multicolumn{3}{|l|}{ Adrenal weight (mg) } \\
\hline Dam & $35.2 \pm 1.2(8)$ & $32.9 \pm 2.8(7)$ \\
\hline Male weanling & $8.41 \pm 0.79(7)$ & $8.91 \pm 0.66(7)$ \\
\hline Female weanling & $7.78 \pm 0.53(8)$ & $7.81 \pm 0.89(7)$ \\
\hline \multicolumn{3}{|l|}{ Testis weight (mg) } \\
\hline Male weanling & $112.9 \pm 6.9(7)$ & $111.0 \pm 6.6(7)$ \\
\hline \multicolumn{3}{|l|}{ Uterus weight (mg) } \\
\hline Dam & $440 \pm 29(8)$ & $610 \pm 77(7)^{*}$ \\
\hline Female weanling & $79.0 \pm 8.2(8)$ & $62.4 \pm 12.6(7)$ \\
\hline
\end{tabular}

Results are expressed as means \pm S.E. The numbers in parentheses refer to the numbers of rats. * Significantly different from the control $(p<0.05)$.

Table 2. Anogenital distance (AGD), vaginal opening and estrus cycle in offspring after exposure to IBP during gestation and lactation

\begin{tabular}{|c|c|c|c|c|c|}
\hline \multirow{2}{*}{ Treatment } & \multicolumn{2}{|c|}{ AGD at 7 days of age } & \multicolumn{2}{|c|}{ Vaginal opening } & \multirow{2}{*}{$\begin{array}{l}\text { Estrous cycle at } 7 \\
\text { weeks of age (day) }\end{array}$} \\
\hline & Male & Female & Age (day) & Body weight (g) & \\
\hline Control & $6.90 \pm 0.10(8)$ & $4.07 \pm 0.11(8)$ & $32.4 \pm 0.5(8)$ & $117.6 \pm 5.1(8)$ & $4.19 \pm 0.09(8)$ \\
\hline IBP & $7.14 \pm 0.11$ & $4.07 \pm 0.17(7)$ & $32.2 \pm 0.7(5)$ & $121.1 \pm 5.2(5)$ & $4.00 \pm 0.00(5)$ \\
\hline
\end{tabular}

Each value is a mean \pm S.E.

corticoids during the second and third week of pregnancy were shown to alter the plasma adrenocorticotropic hormone (ACTH), but not corticosterone, stress responses, especially in males [31]. These results suggest that ACTH release in offspring may be influenced by early exposure to IBP, though the adrenal weight and corticosterone and pro- lactin levels were not influenced in the 12-week-old male offspring with or without immobilization stress or in the male and female weanlings. However, the decrease in the maternal corticosterone level induced by maternal IBP exposure in our study did not affect the thyroid function of the offspring, although a previous study showed that 
Table 3. Organ weights and plasma hormone concentrations in adult female offspring after exposure to IBP during gestation and lactation

\begin{tabular}{|c|c|c|}
\hline & Control & IBP \\
\hline \multicolumn{3}{|c|}{ Plasma hormone concentrations } \\
\hline \multicolumn{3}{|c|}{$\mathrm{LH}(p \mathrm{~g} / \mathrm{m} l)$} \\
\hline Intact $^{\mathrm{a})}$ & $1.76 \pm 0.74(6)$ & $3.96 \pm 3.19(4)$ \\
\hline $\mathrm{OVX}^{\mathrm{b})}$ & $16.52 \pm 2.87(7)$ & $13.46 \pm 2.08(7)$ \\
\hline $\mathrm{OVX}+\mathrm{E} 2^{\mathrm{c})}$ & $0.576 \pm 0.280(5)$ & $0.455 \pm 0.306(5)$ \\
\hline \multicolumn{3}{|l|}{$\mathrm{FSH}(p \mathrm{~g} / \mathrm{m} l)$} \\
\hline OVX & $39.5 \pm 8.2(6)$ & $30.1 \pm 5.6(4)$ \\
\hline $\mathrm{OVX}+\mathrm{E} 2$ & $6.36 \pm 1.17(6)$ & $5.93 \pm 1.12(6)$ \\
\hline \multicolumn{3}{|l|}{$\operatorname{PRL}(p \mathrm{~g} / \mathrm{m} l)$} \\
\hline Intact & $1.73 \pm 0.40(5)$ & $2.35 \pm 1.23(3)$ \\
\hline OVX & $12.18 \pm 10.99(7)$ & $2.77 \pm 1.37(6)$ \\
\hline $\mathrm{OVX}+\mathrm{E} 2$ & $457.3 \pm 197.9(8)$ & $245.5 \pm 77.3(6)$ \\
\hline \multicolumn{3}{|l|}{$\mathrm{E} 2(p \mathrm{~g} / \mathrm{m} l)$} \\
\hline Intact & $19.2 \pm 6.7(6)$ & $20.1 \pm 6.8(5)$ \\
\hline OVX + E2 & $38.2 \pm 12.5(8)$ & $34.6 \pm 7.8(6)$ \\
\hline \multicolumn{3}{|l|}{ Organ weight } \\
\hline \multicolumn{3}{|c|}{ Pituitary weight (mg) } \\
\hline $\mathrm{OVX}+\mathrm{E} 2$ & $30.1 \pm 1.9(8)$ & $23.4 \pm 3.6(5)$ \\
\hline \multicolumn{3}{|c|}{ Uterus weight (mg) } \\
\hline $\mathrm{OVX}+\mathrm{E} 2$ & $588.2 \pm 20.7(8)$ & $510.5 \pm 16.1(6)^{*}$ \\
\hline
\end{tabular}

Results are expressed as means \pm S.E. The numbers in parentheses refer to the numbers of rats. * Significantly different from the control $(p<0.05)$.

a) Seventeen-week-old, diestrus.

b) Thirteen-week-old. OVX: ovariectomized.

c) Twelve-week-old. E2: 17- $\beta$ estradiol.

decrease of the maternal corticosterone level induces low plasma T3 levels in offspring [25].

The weight of the uterus in the estradiol-treated OVX offspring at 12 weeks of age was decreased by IBP exposure during gestation and lactation. This result raises the possibility that there is a decrease in expression of ER and the rate of proliferation in the uterus. Similarly, previously published works show that the ER $\alpha$ expression and rate of proliferation in the uterus of animals treated with other estrogenic compounds are significantly lower than in control animals $[14,30]$ and that $\operatorname{ER} \beta$ expression in the fetal ovaries of animals treated with butyl paraben are significantly lower than in control animals [28]. On the other hand, maternal adrenal insufficiency or alteration of sensitivity to estrogen has been reported to affect anxiety and learning behaviors $[11,31]$; therefore, maternal IBP may affect some behaviors, although maternal IBP exposure did not induce hormonal changes in the present study.

Markers of male reproductive function did not differ between the control and IBP-exposed rats, except for the fact that $\mathrm{BCF}$ was increased in the 14-week-old male rats. Although maternal exposure to 100 and $200 \mathrm{mg} / \mathrm{kg} /$ day of butyl paraben on GD6 to PD20 affects the development of the reproductive organs and the sperm count in F1 offspring at PD90 [13], exposure to $2 \mathrm{mg} / \mathrm{kg}$ /day of butyl paraben on PD2 to PD18 has no detectable effect on testis weight and epithelial cell morphology in the efferent ducts on PD18 [6], and exposure to $400 \mathrm{mg} / \mathrm{kg} /$ day of ethyl paraben, $200 \mathrm{mg} /$ $\mathrm{kg} /$ day and $400 \mathrm{mg} / \mathrm{kg} /$ day of butyl paraben on GD7 to
GD21 has no detectable effect on testosterone production and testicular histopathology on GD21 [28]. In the female offspring, maternal exposure to IBP did not affect vaginal opening, the estrous cycle and the plasma hormone levels in the present study, though a previous study demonstrated that vaginal opening in female offspring was significantly decreased at $100 \mathrm{mg} / \mathrm{kg} /$ day butyl paraben but not at 200 $\mathrm{mg} / \mathrm{kg} /$ day [13]. IBP may not have altered male and female reproductive function in the present study, but the effects of other levels of IBP exposure are unclear. In addition, perinatal exposure to IBP did not affect the ratio of male pups or AGD. This is consistent with recent studies by Taxivig et al. [28] and Kang et al. [13], who observed that exposure to butyl paraben does not affect the ratio of male pups or AGD.

While maternal exposure to IBP decreased the plasma corticosterone concentration and increased the uterus weight in dams and uterine sensitivity to estrogen in adult female offspring, the other indices examined were largely unaffected by the present treatment. Even though these results indicate little sign of endocrine disrupting effects for IBP, the existence of activity may be a matter of concern due to the possible impact on the health of future generations.

ACKNOWLEDGMENTS. We thank Dr. Trisonboon Hataitip and Mrs. Yukiko Okada for assistance with experiments. This work was supported in part by the "High-Tech Research Center" Project for Private Universities: matching fund subsidy from the Ministry of Education, Culture, Sports, Science and Technology (MEXT) of Japan (to T. H.) 
Table 4. Organ weights and plasma hormone concentrations in adult male offspring after exposure to IBP during gestation and lactation

\begin{tabular}{|c|c|c|}
\hline & Control & IBP \\
\hline \multicolumn{3}{|l|}{ Plasma hormone concentrations $^{a)}$} \\
\hline Testosterone $(n \mathrm{~g} / \mathrm{m} l)$ & $231.3 \pm 91.0(7)$ & $234.9 \pm 78.6(7)$ \\
\hline ir-Inhibin $(p \mathrm{~g} / \mathrm{m} l)$ & $1.15 \pm 0.04(7)$ & $1.12 \pm 0.12(6)$ \\
\hline $\mathrm{LH}(p \mathrm{~g} / \mathrm{m} l)$ & $0.298 \pm 0.062(7)$ & $0.171 \pm 0.035(7)$ \\
\hline $\mathrm{FSH}(p \mathrm{~g} / \mathrm{m} l)$ & $3.15 \pm 0.33(7)$ & $6.66 \pm 1.65(7)$ \\
\hline Corticosterone $(n \mathrm{~g} / \mathrm{m} l)$ & $98.6 \pm 17.5(7)$ & $111.9 \pm 34.0(7)$ \\
\hline \multicolumn{3}{|l|}{ Corticosterone $(n \mathrm{~g} / \mathrm{m} l)$} \\
\hline after $2 \mathrm{hr}$ immobilization & $452.5 \pm 56.2(6)$ & $329.7 \pm 58.1(6)$ \\
\hline PRL $(p \mathrm{~g} / \mathrm{m} l)$ & $1.53 \pm 1.03(7)$ & $2.18 \pm 0.88(7)$ \\
\hline \multicolumn{2}{|l|}{ Organ weighta) } & $5.09 \pm 2.48(6)$ \\
\hline Pituitary weight (mg) & $15.1 \pm 1.0(7)$ & $14.1 \pm 0.8(7)$ \\
\hline Testis weight $(\mathrm{g})$ & $1.79 \pm 0.05(7)$ & $1.74 \pm 0.04(7)$ \\
\hline Adrenal weight (mg) & $30.2 \pm 2.9(7)$ & $28.0 \pm 3.7(7)$ \\
\hline \multicolumn{3}{|l|}{ Adrenal weight (mg) after } \\
\hline $2 \mathrm{hr}$ immobilization & $32.9 \pm 3.7(7)$ & $32.2 \pm 3.6(6)$ \\
\hline \multicolumn{3}{|l|}{ Semen analysis ${ }^{b)}$} \\
\hline $\mathrm{SHC}\left(\times 10^{7}\right)$ & $15.99 \pm 2.25(4)$ & $13.82 \pm 1.68(4)$ \\
\hline Motile percent (\%) & $80.5 \pm 2.64(5)$ & $79.0 \pm 1.74(5)$ \\
\hline $\operatorname{VSL}(\mu \mathrm{m} / \mathrm{s})$ & $67.2 \pm 8.62(5)$ & $58.1 \pm 3.90(5)$ \\
\hline $\operatorname{VCL}(\mu \mathrm{m} / \mathrm{s})$ & $267.2 \pm 13.9(5)$ & $263.6 \pm 9.5(5)$ \\
\hline LIN & $2.9 \pm 0.17(5)$ & $2.7 \pm 0.16(5)$ \\
\hline ALH mean $(\mu \mathrm{m})$ & $3.8 \pm 0.15(5)$ & $3.7 \pm 0.10(5)$ \\
\hline $\mathrm{ALH} \max (\mu \mathrm{m})$ & $12.2 \pm 0.40(5)$ & $12.0 \pm 0.34(5)$ \\
\hline $\mathrm{BCF}(\mathrm{Hz})$ & $23.6 \pm 0.5(5)$ & $25.2 \pm 0.3(5)^{*}$ \\
\hline $\mathrm{AR}(\mu \mathrm{m})$ & $30.9 \pm 1.32(5)$ & $29.1 \pm 1.20(5)$ \\
\hline
\end{tabular}

Results are expressed as means \pm S.E. The numbers in parentheses refer to the numbers of rats. * Significantly different from the control $(p<0.05)$.

a) Twelve-week-old.

b) Fourteen-week-old. SHC: Sperm head count. VSL: Straight line velocity. VCL: Curvilinear velocity. LIN: Linearity index. ALH: Amplitude of lateral head displacement. BCF: Beat/ Cross frequency. AR: Average radius.

and a Grant-in Aid for Scientific Research (B-18 310044) from the Japan Society for the Promotion of Science (to K. T.).

\section{REFERENCES}

1 Burgess, L.H. and Handa, R.J. 1992. Chronic estrogen-induced alterations in adrenocorticotropin and corticosterone secretion, and glucocorticoid receptor-mediated functions in female rats. Endocrinology 131: 1261-1269.

2 Cashman, A.L. and Warshaw, E.M. 2005. Parabens: a review of epidemiology, structure, allergenicity, and hormonal properties. Dermatitis 16: 57-66; quiz 55-56.

3 Cooke, P.S., Young, P., Hess, R.A. and Cunha, G.R. 1991. Estrogen receptor expression in developing epididymis, efferent ductules, and other male reproductive organs. Endocrinology 128: 2874-2879.

4 Darbre, P.D. and Harvey, P.W. 2008. Paraben esters: review of recent studies of endocrine toxicity, absorption, esterase and human exposure, and discussion of potential human health risks. J. Appl. Toxicol. 28: 561-578.

5 Fisher, J.S., Millar, M.R., Majdic, G., Saunders, P.T., Fraser, H.M. and Sharpe, R.M. 1997. Immunolocalisation of oestrogen receptor-alpha within the testis and excurrent ducts of the rat and marmoset monkey from perinatal life to adulthood. $J$.
Endocrinol. 153: 485-495.

6 Fisher, J.S., Turner, K.J., Brown, D. and Sharpe, R.M. 1999. Effect of neonatal exposure to estrogenic compounds on development of the excurrent ducts of the rat testis through puberty to adulthood. Environ. Health Perspect. 107: 397-405.

7 Golden, R., Gandy, J. and Vollmer, G. 2005. A review of the endocrine activity of parabens and implications for potential risks to human health. Crit. Rev. Toxicol. 35: 435-458.

8 Hamada, T., Watanabe, G., Kokuho, T., Taya, K., Sasamoto, S., Hasegawa, Y., Miyamoto, K. and Igarashi, M. 1989. Radioimmunoassay of inhibin in various mammals. J. Endocrinol. 122: 697-704.

9 Herath, C.B., Jin, W., Watanabe, G., Arai, K., Suzuki, A.K. and Taya, K. 2004. Adverse effects of environmental toxicants, octylphenol and bisphenol A, on male reproductive functions in pubertal rats. Endocrine. 25: 163-172.

10 Hoberman, A.M., Schreur, D.K., Leazer, T., Daston, G.P., Carthew, P., Re, T., Loretz, L. and Mann, P. 2008. Lack of effect of butylparaben and methylparaben on the reproductive system in male rats. Birth Defects Res. B Dev. Reprod. Toxicol. 83: $123-133$.

11 Imwalle, D.B., Bateman, H.L., Wills, A., Honda, S., Harada, N. and Rissman, E.F. 2006. Impairment of spatial learning by estradiol treatment in female mice is attenuated by estradiol exposure during development. Horm. Behav. 50: 693-698.

12 Jaroenporn, S., Furuta, C., Nagaoka, K., Watanabe, G. and 
Taya, K. 2008. Comparative effects of prolactin versus ACTH, estradiol, progesterone, testosterone, and dihydrotestosterone on cortisol release and proliferation of the adrenocortical carcinoma cell line H295R. Endocrine. 33: 205-209.

13 Kang, K.S., Che, J.H., Ryu, D.Y., Kim, T.W., Li, G.X. and Lee, Y.S. 2002. Decreased sperm number and motile activity on the F1 offspring maternally exposed to butyl p-hydroxybenzoic acid (butyl paraben). J. Vet. Med. Sci. 64: 227-235.

14 Khurana, S., Ranmal, S. and Ben-Jonathan, N. 2000. Exposure of newborn male and female rats to environmental estrogens: delayed and sustained hyperprolactinemia and alterations in estrogen receptor expression. Endocrinology 141: 4512-4517.

15 Korenman, S.G., Stevens, R.H., Carpenter, L.A., Robb, M., Niswender, G.D. and Sherman, B.M. 1974. Estradiol radioimmunoassay without chromatography: procedure, validation and normal values. J. Clin. Endocrinol. Metab. 38: 718-720.

16 Lee, H.C., Yamanouchi, K. and Nishihara, M. 2006. Effects of perinatal exposure to phthalate/adipate esters on hypothalamic gene expression and sexual behavior in rats. J. Reprod. Dev. 52: 343-352.

17 Meistrich, M.L. 1989. Evaluation of reproductive toxicity by testicular sperm head counts. J. Am. Coll. Toxicol. 8: 551-567.

18 Morohoshi, K., Yamamoto, H., Kamata, R., Shiraishi, F., Koda, T. and Morita, M. 2005. Estrogenic activity of 37 components of commercial sunscreen lotions evaluated by in vitro assays. Toxicol. In Vitro 19: 457-469.

19 Oishi, S. 2002. Effects of butyl paraben on the male reproductive system in mice. Arch. Toxicol. 76: 423-429.

20 Oishi, S. 2001. Effects of butylparaben on the male reproductive system in rats. Toxicol. Ind. Health. 17: 31-39.

21 Oishi, S. 2004. Lack of spermatotoxic effects of methyl and ethyl esters of $p$-hydroxybenzoic acid in rats. Food Chem. Toxicol. 42: 1845-1849.

22 Prusakiewicz, J.J., Harville, H.M., Zhang, Y., Ackermann, C. and Voorman, R.L. 2007. Parabens inhibit human skin estrogen sulfotransferase activity: possible link to paraben estro- genic effects. Toxicology 232: 248-256.

23 Rastogi, S.C., Schouten, A., de Kruijf, N. and Weijland, J.W. 1995. Contents of methyl-, ethyl-, propyl-, butyl- and benzylparaben in cosmetic products. Contact Dermatitis 32: 28 30.

24 Routledge, E.J., Parker, J., Odum, J., Ashby, J. and Sumpter, J.P. 1998. Some alkyl hydroxy benzoate preservatives (parabens) are estrogenic. Toxicol. Appl. Pharmacol. 153: 12-19.

25 Slone-Wilcoxon, J. and Redei, E.E. 2004. Maternal-fetal glucocorticoid milieu programs hypothalamic-pituitary-thyroid function of adult offspring. Endocrinology 145: 4068-4072.

26 Takasugi, N. and Bern, H.A. 1964. Tissue changes in mice with persistent vaginal cornification induced by early postnatal treatment with estrogen. J. Natl. Cancer Inst. 33: 855-865.

27 Tavares, R.S., Martins, F.C., Oliveira, P.J., Ramalho-Santos, J. and Peixoto, F.P. 2009. Parabens in male infertility-Is there a mitochondrial connection? Reprod. Toxicol. 27: 1-7.

28 Taxvig, C., Vinggaard, A.M., Hass, U., Axelstad, M., Boberg, J., Hansen, P.R. 2008. Frederiksen, H. and Nellemann, C., Do parabens have the ability to interfere with steroidogenesis? Toxicol. Sci. 106: 206-213.

29 Taya, K., Watanabe, G. and Sasamoto, S. 1985. Radioimmunoassay for progesterone, testosterone and estradiol-17beta using $\mathrm{I}^{125}$ - iodohistamine radioligands. Jpn. J. Anim. Reprod. 31: 186-197.

30 Varayoud, J., Ramos, J.G., Bosquiazzo, V.L., Munoz-de-Toro, M. and Luque, E.H. 2008. Developmental exposure to bisphenol a impairs the uterine response to ovarian steroids in the adult. Endocrinology 149: 5848-5860.

31 Wilcoxon, J.S. and Redei, E.E. 2007. Maternal glucocorticoid deficit affects hypothalamic-pituitary-adrenal function and behavior of rat offspring. Horm. Behav. 51: 321-327.

32 Ye, X., Bishop, A.M., Reidy, J.A., Needham, L.L. and Calafat, A.M. 2006. Parabens as urinary biomarkers of exposure in humans. Environ. Health Perspect. 114: 1843-1846. 